\title{
L'île d'Anjouan figure de la balkanisation de l'archipel des Comores
}

\section{François Taglioni}

\section{(2) OpenEdition}

Journals

Édition électronique

URL : http://journals.openedition.org/echogeo/7223

DOI : $10.4000 /$ echogeo.7223

ISSN : 1963-1197

Éditeur

Pôle de recherche pour l'organisation et la diffusion de l'information géographique (CNRS UMR 8586)

\section{Référence électronique}

François Taglioni, «L'île d'Anjouan figure de la balkanisation de l'archipel des Comores », EchoGéo [En ligne], Sur le Vif, mis en ligne le 02 septembre 2008, consulté le 30 avril 2019. URL : http:// journals.openedition.org/echogeo/7223 ; DOI : 10.4000/echogeo.7223

Ce document a été généré automatiquement le 30 avril 2019.

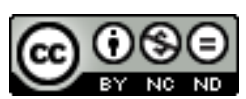

EchoGéo est mis à disposition selon les termes de la licence Creative Commons Attribution - Pas d'Utilisation Commerciale - Pas de Modification 4.0 International 


\title{
L'île d'Anjouan figure de la balkanisation de l'archipel des Comores
}

\author{
François Taglioni
}

\section{Introduction}

1 Le débarquement d'une force internationale à Anjouan le 25 mars 2008 est un nouvel épisode de l'instabilité politique chronique qui règne dans l'Union des Comores depuis son indépendance le 6 juillet 1975. Cette intervention de l'armée comorienne appuyée par des troupes sous l'égide de l'Union africaine a été justifiée par le désir du président de l'Union, M. Ahmed Abdallah Sambi, de mettre un terme aux desseins sécessionnistes du Président d'Anjouan, M. Mohamed Bacar. Cette situation, assez extrême dans le cas des Comores, est caractéristique des difficultés de l'intégration et de l'union politique dans les Etats multi-insulaires où la tentation du vide, autrement dit de se déprendre des autres composantes de l'Etat-nation, est grande. Les motivations de cette balkanisation archipélagique sont complexes et multiples, nous allons nous y attarder dans cet article.

\section{Un archipel marginalisé}

2 L'archipel volcanique, au sens propre et figuré, des Comores ${ }^{i}$ est géographiquement à l'interface de l'Afrique sud-orientale et de Madagascar (cf. Figure 1) et culturellement à la rencontre de mondes aussi divers que l'Afrique, l'Arabie, l'Europe ou encore l'Indonésie et le sud-ouest de l'océan Indien. Néanmoins, l'histoire et la géographie se sont conjuguées pour placer cet archipel en marge du développement politique, économique et des flux mondiaux. L'archipel est constitué de quatre îles, Anjouan, Grande Comore, Mayotte et Mohéli. Cependant, d'un point de vue institutionnel on distingue d'une part l'île de Mayotte, collectivité départementale française et d'autre part l'Union des Comores, Etat indépendant depuis 1975 qui regroupe les trois autres îles. 
3 La situation économique est très difficile dans l'Union des Comores avec peu ou pas d'investissements publics ou privés dans les infrastructures essentielles au développement dans les domaines primordiaux de la santé, de l'éducation, du social, des transports et du tourisme. Autant de secteurs, et il y en a d'autres, qui souffrent de finances publiques très déficitaires. La structure de l'économie reflète largement l'organisation de la société comorienne qui est majoritairement rurale (environ $70 \%$ ) et qui reste amplement tournée vers le secteur primaire (environ $40 \%$ du PIB) avec des cultures de rentes d'exportation concentrées sur la vanille, l'ylang-ylang et le clou de girofle. Le secondaire est embryonnaire et se résume à quelques entreprises agroalimentaires et de manufactures. C'est une industrie de type artisanal comme c'est souvent le cas dans les petits espaces insulaires du Pacifique à la Caraibe en passant par l'océan Indien. Le tertiaire compte pour près de la moitié du PIB du pays mais il ne produit que peu de richesses et s'appuie sur les finances de l'état, elles-mêmes tributaires des aides internationales et d'une dette très lourde (environ $70 \% \mathrm{du}$ PIB). Une autre source importante de revenu pour le pays est constituée par les transferts de

4 fonds de la diaspora comorienne établie en France métropolitaine et d'outre-mer, avec une très forte concentration dans la ville de Marseille ${ }^{\mathrm{ii}}$, à Mayotte, à La Réunion et à Madagascar. L'apport de la diaspora, forte de plusieurs milliers de personnes qui transfèrent environ 20 milliards de francs comoriens (40 de millions d'euros) vers l'Union, sert à financer le budget alimentaire, la santé et l'éducation mais il n'est pas suffisant pour assurer un décollage économique. En effet, cet argent est souvent insuffisamment valorisé par les Comoriens restés au pays.

Figure 1 - Le Sud-Ouest de l'océan Indien

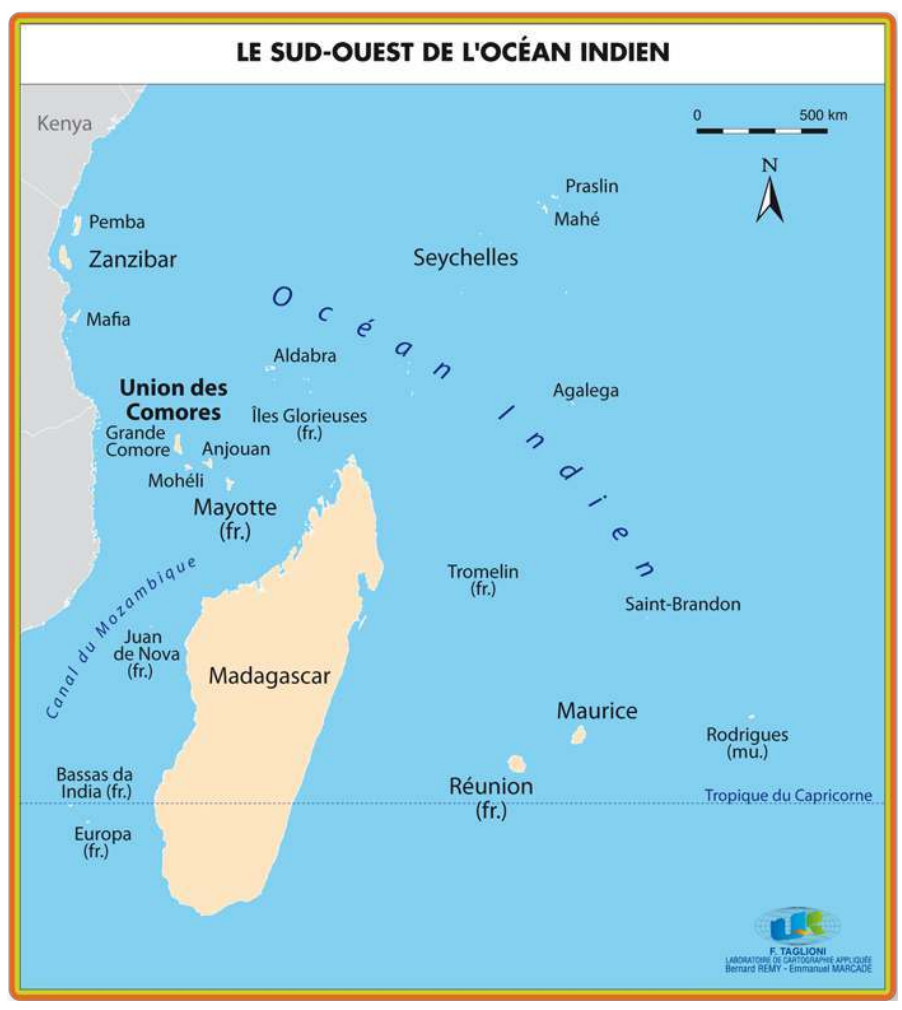

5 On le voit, le mode de fonctionnement économique de l'Union des Comores est assez conforme au modèle MIRAB, développé dans les années 1980 par Bertram et Watters 
(Bertram \& Watters, 1986). L'équation du MIRAB est la suivante : Mi pour migration ( migration) ; R pour envois de fonds (remittances) ; A pour financement par l'aide (aid financed) et $B$ pour bureaucratie (Bureaucracy). Ce modèle montre que les petits espaces insulaires du Pacifique sont fortement tributaires de ces quatre composantes. En fait, et dans une certaine mesure, ce modèle est transposable à la majorité des petits espaces insulaires en développement et notamment aux Comores.

Le développement humain dans l'Union des Comores est globalement fragile (cf. Figure 2) avec par exemple une mortalité infantile élevée, un taux d'alphabétisation inférieur à 60 $\%$ et une espérance de vie assez limitée pour ne prendre que quelques indicateurs. Ces résultats traduisent que près de $50 \%$ de la population vit sous le seuil de pauvreté. Ils sont d'autant plus inquiétants que le PIB par habitant à prix constants était supérieur à la fin des années $1920^{\text {iii }}$ à celui d'aujourd'hui. Il s'établissait en 1929 à 350 dollars par habitant contre 330 dollars par habitant en 1999.

Figure 2 - L'archipel des Comores en quatre chiffres

\begin{tabular}{|c|c|c|c|c|}
\hline & $\begin{array}{c}\text { Superficie } \\
\mathbf{k m}^{2}\end{array}$ & $\begin{array}{c}\text { Population } \\
\text { estimée }\end{array}$ & $\begin{array}{c}\text { Mortalité infantile } \\
\text { estimée } \\
\text { en \%o }\end{array}$ & $\begin{array}{c}\text { Espérance de vie } \\
\text { estimée } \\
\text { en année }\end{array}$ \\
\hline Anjouan & 424 & 250000 & & \\
\cline { 1 - 3 } Grande Comore & 1148 & 365000 & \multirow{2}{*}{59} & 63 \\
\cline { 1 - 3 } Mohéli & 290 & 32000 & 15 & 75 \\
\hline Mayotte & 374 & 186000 & 15 & 75 \\
\hline
\end{tabular}

Source : Ined, Insee, Commissariat général du plan de l'union des Comores

7 La situation est très sensiblement différente à Mayotte où, si l'on s'en tient à quelques indicateurs, le développement humain est beaucoup plus élevé que dans le reste de l'archipel. Alors que l'Union des Comores stagne voire régresse économiquement, Mayotte continue de connaître une embellie de son niveau de vie et de ses infrastructures de développement. La collectivité départementale n'a pas encore atteint les standards de sa voisine, La Réunion, qui jouit d'un statut de département, ni de celui de la métropole, mais elle est néanmoins très attractive pour les Comoriens qui, nous l'avons dit, vivent dans des conditions globalement très précaires. Cette différence de développement, directement imputable au statut de collectivité départementale et aux transferts de fonds français et européens, engendre notamment une forte pression migratoire (cf. Figure 3) de l'Union des Comores sur Mayotte. On estime ainsi à environ 55000 les Comoriens clandestins à Mayotte, soit un quart de la population mahoraise.

Dans ces flux de migrants comoriens à destination de Mayotte, l'île d'Anjouan joue un rôle particulier. Elle se présente en effet, en raison de sa position géographique la plus proche de Mayotte, comme un « hub », sorte de passerelle entre les trois îles de l'Union et l'eldorado supposé que symbolise la collectivité française. Par ailleurs, Anjouan est la deuxième île la plus peuplée de l'Union mais surtout la plus pauvre et c'est donc elle qui fournit le plus gros des bataillons clandestins. Enfin, les relations familiales et la proximité culturelle lient très fortement les rives d'Anjouan à celles de Mayotte. Il n'est pas une famille mahoraise qui n'ait un parent ou un proche à Anjouan où dans les deux autres îles de l'Union. Les clandestins tentent leur chance de passage dans des embarcations de fortune, appelées Kwassa kwassa. Ce mode d'immigration de boat people du sud-ouest de l'océan Indien est extrêmement dangereux et l'on estime que plusieurs dizaines de personnes périssent chaque année par noyade durant la traversée des 75 
kilomètres qui séparent Anjouan de Mayotte. Ce drame humain se poursuit dans une certaine mesure à Mayotte où les Comoriens sont traités en citoyens de deuxième zone, où ils sont très souvent exploités par des patrons peu scrupuleux et où ils provoquent chez les Mahorais des sentiments de rejet pour ne pas dire de xénophobie.

Figure 3 - L'immigration comorienne vers Mayotte

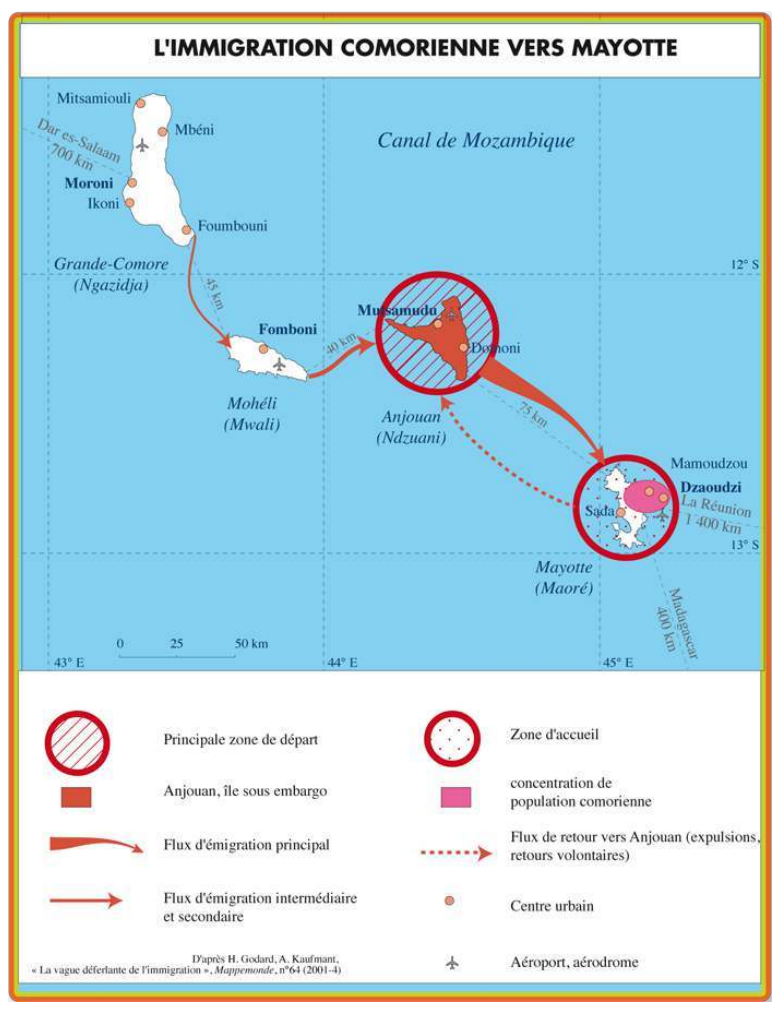

On est finalement entre deux mondes que sépare une frontière maritime sujette à caution. Et c'est justement sur cette notion de frontière que le cas de l'archipel des Comores devient singulier et qu'il nous entraîne sur un processus de balkanisation commencé, il y a plus de 30 ans.

\section{Un processus de balkanisation omniprésent}

La différence de statut entre les membres de l'archipel, avec Mayotte d'un côté et les trois îles de l'Union de l'autre, est dénoncée depuis l'indépendance en 1975 par l'Union des Comores, l'Organisation des nations unies (ONU) et l'Union africaine (UA) qui considèrent que la décolonisation est inachevée puisque qu'une des entités de l'archipel ne fait pas partie de l'Union. Mayotte avait en effet dit «non» à plus de $60 \%$ des votants, le 22 décembre 1974, au premier référendum d'autodétermination des Comores. Lors d'un deuxième référendum le 8 février 1976, Mayotte réitère avec force et vigueur son souhait, à plus de $99 \%$ des votants, de demeurer au sein de la République Française. S'agissant de la première consultation du 22 décembre 1974, on peut s'étonner que le processus d'accession à l'indépendance de l'archipel ne soit pas mis en oeuvre sur la base d'une consultation globale, comme prévu par un accord franco-comorien du 15 juin 1973, mais, au contraire, île par île. Ensuite, les motivations profondes des Mahorais à rester dans le giron de la France sont toujours controversées car il semble bien que des meneurs 
d'opinions aient entretenu sur l'île la peur et les rumeurs à propos d'un régime autoritaire et arbitraire qui serait mis en place par le premier président des Comores, Ahmed Abdallah, au détriment de Mayotte.

On retrouve ici, les limites de la multi-insularité (Taglioni, 2005) où les rivalités entre les îles, soit pour avoir le leadership sur les autres, soit de peur justement de ce leadership, génèrent des mouvements de replis identitaires très forts qui peuvent aller jusqu'à refuser la constitution de l'Etat ou encore à prendre son autonomie ou son indépendance vis-à-vis de cet Etat s'il est déjà constitué.

La conséquence de cette indépendance à la carte, conclue de façon bilatérale, est que, du point de vue du droit international, la France occupe illégalement l'île de Mayotte qui est considérée comme partie intégrante de l'Union des Comores. En suivant ce raisonnement, l'Union des Comores estime que les habitants des îles d'Anjouan, Grande Comore ou Mohéli qui se rendent à Mayotte ne font que gagner une partie du territoire de l'Union. La migration considérée comme illégale par la France ne serait donc qu'un déplacement de population à l'intérieur d'un pays souverain. Si on pousse encore un peu cette logique, les reconduites à la frontière de plusieurs dizaines de Comoriens chaque année vers Anjouan peuvent être considérées comme des transferts forcés de population, c'est en tout cas comme cela que le gouvernement de l'Union le présente.

13 C'est donc bien en cela que le cas de l'archipel des Comores diffère des exemples évoqués plus haut : il n'y a pas, au regard de la convention du droit de la mer, de frontières maritimes entre Mayotte et les trois autres îles de l'archipel pas plus que de mer territoriale sous souveraineté française. C'est d'ailleurs depuis seulement 1995 que la France a mis en place un visa, appelé « visa Balladur », obligatoire pour les ressortissants Comoriens qui veulent entrer à Mayotte.

14 Cette séparation de Mayotte du reste de l'Union est le point de départ d'une instabilité politique chronique qui caractérise l'archipel. Il est d'usage d'avancer le nombre d'une vingtaine de coups d'État plus ou moins aboutis quand on évoque l'histoire politique récente des Comores. Nous n'y reviendrons pas car cela est bien décrit par de nombreux articles (et notamment Ibrahime ; Ouledi, 2007). En revanche, les événements récents qui se sont déroulés sur l'île d'Anjouan depuis 2007 illustrent bien les obstacles de cohésion nationale de l'Etat multi-insulaire des Comores.

\section{Anjouan la rebelle : chronique d'un président déchu}

Mayotte, on vient de le voir, est une des causes importante de la balkanisation de l'archipel des Comores. L'île d'Anjouan est au sein de l'Union une autre force centrifuge qui alimente la destabilisation des institutions de l'Union. Nous n'allons pas retracer l'histoire d'Anjouan depuis l'indépendance des Comores mais simplement revenir sur les événements récents qui ont conduit au débarquement du 25 mars 2008. Pour cela un zoom arrière sur l'année 1997 est nécessaire.

16 En août 1997 Anjouan et Mohéli mettent la pression sécessionniste sur la Grande Comore et elles sortent unilatéralement de la République fédérale islamique des Comores (RFIC était le nom officiel du pays à cette époque). Les choses rentrent vite dans «l'ordre » à Mohéli qui réintègre dès 1998 la RFIC. En revanche, pour Anjouan une nouvelle période de troubles s'amorce avec des affrontements armés entre clans séparatistes qui entraînent des dizaines de morts, un débarquement raté de l'armée comorienne, un embargo et de la 
misère pour les plus démunis. Cette situation va perdurer de 1997 à 2000 où un accord de réconciliation nationale (accord de Fomboni du nom de la capitale de Mohéli) va être signé le 26 août entre les trois îles pour définir les fondements constitutionnels de la nouvelle Union des Comores. Depuis le 17 février 2001 les trois îles ont désormais une très large autonomie et seuls les pouvoirs régaliens (impôts, police, justice, armée) restent du ressort du président de l'Union. En dépit de ce modus vivendi entre les trois composantes de l'Union, la tentation séparatiste est toujours bel et bien là à Anjouan et elle s'incarne dans son président M. Mohamed Bacar.

M. Mohamed Bacar entre en scène politique par un coup d'Etat le 9 août 2001 qui sera entériné et formalisé le 31 mars 2002. Son premier mandat présidentiel de 2002 à 2007 est marqué par une récession économique, une baisse générale du développement humain et un accroissement de la corruption au profit notamment de l'équipe présidentielle et du président. Durant cette période, M. Mohamed Bacar agite en permanence, comme une provocation en direction de Moroni, le spectre de l'indépendance pour Anjouan; la France soutient manifestement les actions de M. Bacar. En juin 2007, M. Mohamed Bacar est réélu, en dépit de la désapprobation du gouvernement central de l'Union des Comores, à la présidence de l'île d'Anjouan. Cette élection ne sera pas non plus reconnue par l'Union africaine et par la communauté internationale. L'élection de M. Ahmed Abdallah Sambi le 14 mai 2006 à la présidence de l'Union va accélérer la chute de M. Bacar. M. Sambi s'est en effet fixé comme objectif prioritaire de restaurer l'union politique entre les trois îles.

18 Ainsi commença le bras de fer entre le gouvernement central de Moroni et le président d'Anjouan. La voie diplomatique ayant échoué, c'est la force armée qui fut choisie pour faire plier Bacar. Le 24 mars 2008 une force internationale composée de militaires de l'Union africaine, principalement des Libyens, des Sénégalais, des Soudanais, des Tanzaniens et bien sûr des Comoriens débarque sur l'île. La France joue officiellement un rôle discret, limitant ses actions aux transports de troupes. Ce débarquement s'effectua sans problème tant la résistance des fidèles à Bacar resta réduite. Il suffit d'un jour pour que Mutsamudu, capitale d'Anjouan, soit aux mains de la coalition "Démocratie aux Comores ». Le 27 mars, M. Bacar, qui a réussi à prendre la fuite, accoste à Mayotte avec 22 de ses proches. La pression monte ainsi à Mayotte où des Anjouanais s'en prennent aux résidents mahorais et métropolitains pour marquer leur désarroi de voir s'enfuir $M$. Bacar.

19 Pour essayer de calmer les esprits, les autorités françaises transfèrent M. Bacar le jour même à La Réunion et l'assigne à résidence avec ses hommes sur une base militaire. Cela n'empêchera pas que Mayotte connaisse plusieurs jours de violence entre communautés ce qui souligne, si besoin en était, que l'histoire a raison de la géographie et que Mayotte s'éloigne inexorablement des îles sœurs de l'Union des Comores. Par ailleurs, la présidence de l'Union des Comores estime que la France a aidé le président déchu à fuir vers Mayotte puis vers La Réunion pour échapper à la justice comorienne. Il semble bien que la France tire toujours les ficelles de la gouvernance de l'archipel. Les raisons sont sans doute stratégiques avec le contrôle du Canal de Mozambique qui assure le passage de nombreux navires et plus particulièrement les pétroliers provenant du Moyen-Orient ou les portes containers asiatiques. Elles sont aussi économiques avec notamment la Zone économique exclusive (ZEE) non négligeable que représente Mayotte et les îles éparses (Europa, Bassas da India, Juan de Nova, Les Glorieuses et Tromelin). 
part, la désunion politique des Comores est sans doute le meilleur moyen de limiter la revendication de Moroni sur Mayotte. L'union faisant la force, il est certain qu'une cohésion entre les membres de la fédération des Comores serait de nature à renforcer une demande légitime de retour de Mayotte dans l'Etat comorien. De ce point de vue, il est manifeste que la France trouve son compte dans l'instabilité chronique de l'Union des Comores.

21 Finalement, et après de nombreux rebondissements, M. Bacar peut maintenant souffler car il sait depuis le 24 juin 2008 qu'il ne sera finalement pas extradé vers Moroni, en dépit d'un procès médiatique ou Me Vergès a soutenu sa demande d'extradition pour le compte du gouvernement central de l'Union des Comores. Il n'obtient cependant pas l'asile politique en France. Il est donc parti en exil au Bénin le samedi 19 juillet 2008 avec trois de ses hommes. Cette demi-mesure de la justice française permet de trouver une solution qui sauve vraisemblablement la vie de M. Bacar, que la France a formé et soutenu pendant des années, et qui permet de maintenir des relations diplomatiques décentes avec le président de l'Union des Comores.

élections ont été organisées à Anjouan en juin 2008. Le deuxième tour du scrutin présidentiel, le 29 juin 2008, a donné la victoire à M. Moussa Toybou qui est un proche du président de l'Union M. Abdallah Sambi. M. Sambi accroît sans doute ainsi son autorité et son ambition politique sur l'archipel.

Cette propension de l'archipel des Comores à l'instabilité politique est un cas d'école. Mais au fond quelles sont les motivations de Mohéli et surtout d'Anjouan pour refuser de vivre au sein d'une fédération politique organisée autour de trois îles? On a longtemps avancé que le frein principal était le ressentiment des hommes politiques locaux à l'encontre de la Grande Comore et de sa capitale Moroni qui était perçue comme hégémonique sur les plans politiques et administratifs. Cela est sans doute vrai, mais c'est à nuancer car la constitution ${ }^{\text {iv }}$ de 2001, qui garantit une grande autonomie à chacune des trois îles de l'Union, n'a pas empêché la montée en puissance indépendantiste d'Anjouan orchestrée par M. Bacar. A ce sentiment typiquement insulaire de revendiquer des particularismes à aucun autre pareil qui se traduisent souvent par un rapport complexe à l'altérité (Taglioni, 2009) se surimpose à Anjouan, comme sur les trois autres îles, plusieurs niveaux de gouvernance en fonction des droits (droit musulman, droit occidental et droit coutumier) et de l'échelle sociale de référence (le village, le clan, la famille). La route de la construction de la nation sera sans doute encore longue pour véritablement parler de l'Union des Comores au sens de la cohésion identitaire d'une nation.

\section{Conclusion}

Dans l'archipel des Comores se superposent de nombreux facteurs qui concourent à la difficile mise en place d'un Etat-nation stable et durable. La société multi-insulaire est stratifiée en fonction de plusieurs influences culturelles, religieuses, historiques ou encore juridiques et politiques. Ce pluralisme de situations se traduit par une instabilité des institutions qui a des répercussions lourdes sur le développement humain freiné depuis plusieurs décennies dans les trois îles de l'Union des Comores. Cette union politique est justement la limite structurelle sur laquelle buttent les différents gouvernements depuis l'indépendance. A ces difficultés endogènes s'ajoute la délicate 
coopération entre l'Union et l'île de Mayotte qui continue d'alimenter un lourd différend politique franco-comorien.

Les événements récents à Anjouan sont là pour rappeler à la présidence de l'Union que l'unité et la stabilité politique, pourtant indispensables entre les trois îles, ne pourront se faire que dans le respect des particularismes des uns et des autres. Ces événements mettent par ailleurs en lumière l'indispensable besoin de clarification de la politique étrangère française vis-à-vis de l'Union. La France ne peut pas espérer endiguer le flot de migrants vers Mayotte en installant seulement de nouveaux radars de surveillance ou en affrétant de nouvelles frégates pour repousser les kwassa kwassa. C'est par une véritable réconciliation et un renouveau de la coopération entre l'Union des Comores, la France et les autres bailleurs de fonds que des solutions pragmatiques pourront voir le jour afin que la pression migratoire sur Mayotte se relâche et qu'une coopération choisie se développe au sein de l'Union.

\section{BIBLIOGRAPHIE}

Artaud, D. et alii, 2004. Mayotte, population et conditions de vie. Economie de La Réunion, $\mathrm{n}^{\circ} 119$, p. $11-22$

Blanchy, S., 2004. Cités, citoyenneté et territorialité dans l'île de Ngazidja (Comores). Journal des africanistes, $n^{\circ} 1 \& 2$, p. $341-380$

Bertram, I. ; Watters, R., 1986. The Mirab process : earlier analyses in context. Pacific Viewpoint, $\mathrm{n}^{\circ} 1$, p. $47-59$

Caminade, P., 2004. Comores-Mayotte, une histoire néo-coloniale. Marseille, Agone éditeur, 188 p. Dépouillement des numéros de mars 2008 à juillet 2008 :

- des trois journaux quotidiens de l'île de La Réunion : Le Journal de l'île de La Réunion; Le Quotidien; Témoignages

- de deux hebdomadaires de Mayotte : Le Mahorais ; Mayotte hebdo

- de deux hebdomadaires de l'Union des Comores : Al Watwan ; Comores-infos

Godard, H. ; Kaufmant, A., 2001. La vague déferlante de l'immigration. MappeMonde, $\mathrm{n}^{\circ} 64$, p. $7-10$

Ibrahime, M. ; Ouledi, A., 2007. Les Comores au jour le jour. Moroni, Komedit, 122 p.

Lacoste, Y., 1991. Balkans et balkanisation. Hérodote, n 63, p. 3-13

Taglioni, F., 2009. Altérité et identité dans les sociétés insulaires : du multiculturalisme stratifié créole à l'éthno-nationalisme fidjien. À paraître dans un ouvrage collectif sous la direction de J.F. Staszak et P.-M. Decoudras

Taglioni, F., 2006. Les petits espaces insulaires face à la variabilité de leur insularité et de leur statut politique. Les Annales de géographie, n652, p. 664-687

Taglioni, F., 2005. Les revendications séparatistes et autonomistes au sein des États et territoires mono- et multi-insulaires. Essai de typologie. Cahiers de géographie du Québec, $n^{\circ} 136$, p. 5-18 
http://www.cgq.ulaval.ca/textes/vol_49/no136/03-Taglioni.pdf

Todorova, M., 1997. Imagining the Balkans. New York, Oxford University Press, 257 p.

Walker, I., 2007. What came first, the nation or the state? Political process in the Comoro Islands. Africa, $n^{\circ} 4$, p. 582-605

\section{NOTES}

i. Une interview radiophonique de l'auteur sur l'archipel des Comores est accessible à cette adresse http://publi.taglioni.net/RCF2008.mp3

ii. On estime à 50000 (mais sans doute beaucoup plus d'après les associations de Comoriens de la capitale phocéenne) le nombre de Comoriens installés à Marseille soit plus de personnes qu'à Moroni capitale politique de l'Union des Comores

iii. Source : Commissariat général du plan de l'union des Comores)

iv. En fait on devrait écrire «les constitutions " puisque chaque île à la sienne et qu'il en existe une au niveau de l'Union des Comores

\section{RÉSUMÉS}

L'Union des Comores connaît une instabilité politique chronique depuis son indépendance en 1975. De tentatives de coups d'état en coups d'état aboutis, de révisions constitutionnelles en réconciliations nationales, l'Union est fragile et essaye de résister à une balkanisation devenue problématique. Cette tendance s'incarne dans l'appartenance politique de la quatrième île de l'archipel des Comores, Mayotte, à la République française et dans les tentatives séparatistes d'Anjouan et Mohéli. C'est dans ce contexte politique tendu qu'a eu lieu le 25 mars 2008 à Anjouan le débarquement d'une force internationale sous l'égide de l'Union africaine pour renverser le président Mohamed Bacar. L'Union des Comores illustre bien les obstacles de cohésion nationale dans un Etat multi-insulaire en prise avec de lourds problèmes politiques sur fond de mal développement économique et humain persistant.

Union of the Comoros is experiencing chronic political instability since its independence in 1975. From coups attempts to coup, from constitutional developments to national reconciliation, the Union is fragile and tries to resist with an endemic balkanization. It is embodied with the political affiliation of the fourth island of the Comoros archipelago, Mayotte, in the French Republic and with the separatist attempts of Anjouan and Moheli.In this tense political context occurred on March 25, 2008 the landing in Anjouan of an international force under the aegis of the African Union to overthrow President Mohamed Bacar. Union of the Comoros illustrates the obstacles of a national cohesion within a multi-insular state wrestling with heavy political difficulties and persistent economic and human development problems. 
INDEX

Mots-clés : Anjouan, archipel des Comores, balkanisation, Mohamed Bacar, multi-insularité

Keywords : Comoros archipelago, multi-insularity

\section{AUTEUR}

\section{FRANÇOIS TAGLIONI}

François Taglioni (tagli@diplomacy.edu) est directeur de recherche à l'IRD, UR 029, en affectation à La Réunion et membre de l'UMR PRODIG. Spécialiste des petits espaces insulaires en développement dans le monde, il travaille actuellement sur l'analyse de la crise provoquée par l'épidémie du chikungunya dans l'océan Indien sous ses aspects à la fois politiques, institutionnels et sociaux.

Site Internet de l'auteur : http://www.taglioni.net 